\title{
Androgen receptor splice variant 7 in castration resistant prostate cancer treated with hormonal therapy: what is in the pipeline?
}

\author{
Martina Chirra ${ }^{1}$, Roberto Petrioli ${ }^{1}$, Giandomenico Roviello ${ }^{2}$ \\ ${ }^{1}$ Department of Oncology, Medical Oncology Unit, University of Siena, Siena, Italy; ${ }^{2}$ Department of Health Sciences, University of Florence, \\ Florence, Italy \\ Correspondence to: Giandomenico Roviello, MD, PhD. Department of Health Sciences, University of Florence, Viale Pieraccini, 6, 50139 Florence, \\ Italy. Email: giandomenicoroviello@hotmail.it. \\ Comment on: Armstrong AJ, Halabi S, Luo J, et al. Prospective Multicenter Validation of Androgen Receptor Splice Variant 7 and Hormone Therapy \\ Resistance in High-Risk Castration-Resistant Prostate Cancer: The PROPHECY Study. J Clin Oncol 2019;37:1120-9.
}

Submitted Aug 13, 2019. Accepted for publication Aug 21, 2019.

doi: $10.21037 /$ tcr.2019.08.34

View this article at: http://dx.doi.org/10.21037/tcr.2019.08.34

Prostate cancer (PC) represents the second most commonly diagnosed cancer in men (1).

Localized PC is more frequently diagnosed than metastatic PC (2). However, the survival of PC is strictly dependent on the staging, with a 5 -year survival rate for the localized disease $>99 \%$, and a 5 -year survival rate for metastatic PC around 30\% (2). Management of metastatic disease remains challenging, both in the hormone-dependent setting and in castration-resistant PC (CRPC), due to the development of resistance and disease progression to the available therapeutic options. FDA has recently approved several drugs for metastatic CRPC (mCRPC): docetaxel, cabazitaxel, androgen receptor (AR) signaling inhibitors (abiraterone acetate and enzalutamide), sipuleucel-T and radium-223. However, the optimal treatment sequence is still unknown, and the complete identification of patients who could benefit the most by each therapy remains an unmet need. In this view, the validation of objective and measurable biomarkers that correlate with the response to a specific therapy is of utmost importance (3).

Numerous mechanisms of resistance have been described along with AR signaling inhibitors, including AR splice variants (AR-Vs), amplification or mutations of the AR gene, enhancement of AR transcriptional activity or stability, loss of AR, increased steroidogenesis, EGR gene rearrangement and upregulation of alternative signaling pathways (4).

In particular, AR-Vs represent abnormal AR proteins, lacking the AR C-terminal ligand-binding domain, but retaining the transactivating $\mathrm{N}$-terminal domain. Due to these characteristics, AR-Vs are constitutively active, promoting AR activity in the absence of the ligand (5). Several AR-Vs have been identified, and the AR splice variant 7 (AR-V7) represents the most promising and biologically significant marker in PC.

Originally, the pretreatment status of AR-V7 was explored in circulating tumor cells (CTCs) of 62 patients affected by mCRPC undergoing abiraterone acetate or Enzalutamide (6). Quantitative reverse-transcriptasepolymerase-chain-reaction (RT-PCR) assays were used for AR-V7 mRNA detection in CTCs. AR-V7 was associated with primary resistance to AR signaling inhibitors. Indeed, AR-V7 positive patients who received abiraterone acetate or Enzalutamide had lower prostate-specific antigen (PSA) response rates, shorter PSA progression-free survival (PFS), clinical/radiographic PFS and overall survival (OS) than AR-V7 negative patients. Interestingly, the negative predictive role of AR-V7 was not confirmed in patients affected by mCRPC undergoing taxane chemotherapy (7). Indeed, AR-V7 status did not influence the response to chemotherapy in this setting, with no significant difference in terms of PSA response rate, PSA PFS and clinical/ radiographic $\mathrm{PFS}$ in AR-V7 positive versus negative patients. However, although AR-V7 was not associated with primary resistance to taxanes, it has been shown that ARV7 positive patients experienced a significant improvement in PSA responses and PFS when received taxanes instead of AR signaling inhibitors. Conversely, AR-V7 negativity 
did not predict a specific benefit by either therapy, with no significant differences between taxanes and AR signaling inhibitors concerning any clinical outcomes in AR-V7 negative patients (7).

Armstrong and colleagues have recently explored the role of AR-V7 in CTCs from 118 patients affected by highrisk $\mathrm{mCRPC}$ and treated with AR signaling inhibitors (8). More in details, in this prospective and multicenter study, the analysis of AR-V7 status on CTCs was performed simultaneously using two different techniques: Epic Sciences (Epic; San Diego, CA, USA) and Johns Hopkins University (JHU; Baltimore, MD, USA) CTC AR-V7 assays. The first (Epic) was a nuclear-specific AR-V7 protein assay; the second (JHU) was an mRNA assay. Both detect the presence of AR-V7. The concordance between the two CTCs assays was $82 \%$, with a percentage of $9 \%$ to $24 \%$ of AR-V7 positive patients at baseline, according to the different technique. The role of AR-V7 was investigated by the authors in terms of PFS, OS, radiographic (per RECIST version 1.1 ) and biochemical (50\% or greater PSA decline) response rate to abiraterone and/or enzalutamide in highrisk mCRPC. After a median follow-up of 19.6 months, median PFS (mPFS) and OS (mOS) were remarkably longer in $\mathrm{AR}-\mathrm{V} 7$ negative compared to $\mathrm{AR}-\mathrm{V} 7$ positive patients with both CTC assays (Epic: mPFS 6.1 versus 3.1 months, mOS 25.5 versus 8.4 months, in AR-V7 negative and AR-V7 positive, respectively; JHU: mPFS 6.9 vs. 3.1 months, mOS 27.2 vs. 10.8 months, in AR$\mathrm{V} 7$ negative and AR-V7 positive, respectively). In short, AR-V7 negativity tested by either Epic or JHU represented a favorable factor. Of note, the prognostic and predictive significance of AR-V7 was independent of CTCs heterogeneity (that has been correlated with worse OS in patients treated with AR signaling inhibitors) (9), CTCs number and Halabi risk score (10), strengthening the connection between AR-V7 positivity and treatment resistance (8). Essentially, through this multicenter study, the authors have prospectively validated the role of AR-V7 as a biomarker of resistance to AR signaling inhibitors in mCRPC, exploring at two independent assays for the ARV7 analysis-Epic and JHU. Despite different rates of ARV7 positive patients with the two techniques, the negative predictive role of $\mathrm{AR}-\mathrm{V} 7$ was confirmed by either assay. Of note, $0 \%$ of patients tested as negative by Epic showed had a RECIST or PSA response, being the assay more specific with no false-positive patients. On the contrary, JHU detected more AR-V7 positive patients but resulting in $6 \%$ to $11 \%$ of $\mathrm{AR}-\mathrm{V} 7$ positive men with confirmed
radiographic/PSA responses. AR-V7 negative patients have more probability to clinically benefit from AR signaling inhibitors compared to AR-V7 positive patients. However, AR-V7 negative men may not respond to abiraterone or enzalutamide, confirming the existence of several mechanisms of resistance independent of AR-V7. In fact, only $25 \%$ of the AR-V7 negative patients by either assay had a radiographic and/or biochemical response.

As mentioned by the authors, future perspectives may imply (I) larger sample size, mainly to increase the number of AR-V7 positive patients; (II) inclusion of different risk groups, exploring the role of AR-V7 not only in highrisk mCRPC; (III) validation of $\mathrm{AR}-\mathrm{V} 7$ as a biomarker of response to taxane chemotherapy in mCRPC; (IV) understanding the pathogenic mechanism responsible for AR-V7-related hormone therapy resistance, with the aim of developing new therapeutic options. Ultimately, one should not forget that the status of AR-V7 might be explored using different methods and biological samples, namely the tumor and the blood through liquid biopsies (3). Liquid biopsies allow detecting AR-V7 in several ways, analyzing cell-free RNA, exosomal RNA, and CTCs-derived protein/ mRNA. In this view, it is of utmost importance to study the reproducibility and clinical implications of the results obtained with different techniques, to validate a biomarker as disease-modifying medical decisions. Moreover, some authors have combined the detection of AR-V7 with other features to better define the resistance to hormone therapy in mCRPC. A recent retrospective study has been published on the prognostic and predictive role of AR-FL-the full length of AR-and AR-V7 (11). The authors analyzed the $\mathrm{AR}-\mathrm{FL}$ and AR-V7 collected from plasma-derived exosomal RNA of 73 patients affected by mCRPC, prior to the beginning of Abiraterone acetate or Enzalutamide. The results of this study confirm the resistance to AR-directed therapy in $\mathrm{AR}-\mathrm{V} 7$ positive patients. Of note, stratifying patients according to AR-FL expression (low, intermediate, and high expression), PFS was inversely correlated with the quantity of AR-FL. Additionally, AR-V7 positive patients with low AR-FL expression had a better outcome in terms of PFS compared to intermediate and high ARFL expression. In this view, the availability of both ARFL and AR-V7 status could predict the response to AR signaling inhibitors. In addition, Antonarakis et al. (12) have investigated the outcome of patients with mCRPC undergoing Abiraterone acetate or Enzalutamide according to three separate prognostic categories: CTC-, CTC+/ AR-V7-, and CTC+/AR-V7+. The outcome was worst for 
$\mathrm{CTC}+\mathrm{AR}-\mathrm{V} 7+$ patients, intermediate for $\mathrm{CTC}+\mathrm{AR}-\mathrm{V} 7$ patients and best for CTC- patients.

In conclusion, $\mathrm{AR}-\mathrm{V} 7$ is a promising biomarker in mCRPC, even if further efforts are required to thoroughly validate its role. Although the road seems still long, scientific advance runs fast, and the establishment of an accurate predictive model in $\mathrm{mCRPC}$ is closer than we would have expected.

\section{Acknowledgments}

Funding: None.

\section{Footnote}

Provenance and Peer Review: This article is commissioned and reviewed by the Section Editor Dr. Peng Zhang (Department of Urology, Zhongnan Hospital of Wuhan University, Wuhan, China).

Conflicts of Interest: All authors have completed the ICMJE uniform disclosure form (available at http://dx.doi. org/10.21037/tcr.2019.08.34). The authors have no conflicts of interest to declare.

Ethical Statement: The authors are accountable for all aspects of the work in ensuring that questions related to the accuracy or integrity of any part of the work are appropriately investigated and resolved.

Open Access Statement: This is an Open Access article distributed in accordance with the Creative Commons Attribution-NonCommercial-NoDerivs 4.0 International License (CC BY-NC-ND 4.0), which permits the noncommercial replication and distribution of the article with the strict proviso that no changes or edits are made and the original work is properly cited (including links to both the formal publication through the relevant DOI and the license). See: https://creativecommons.org/licenses/by-nc-nd/4.0/.

\section{References}

1. Bray F, Ferlay J, Soerjomataram I, et al. Global cancer statistics 2018: GLOBOCAN estimates of incidence and mortality worldwide for 36 cancers in 185 countries. CA Cancer J Clin 2018;68:394-424.

2. Siegel RL, Miller KD, Jemal A. Cancer statistics, 2019. CA Cancer J Clin 2019;69:7-34.
3. Bastos DA, Antonarakis ES. CTC-derived AR-V7 detection as a prognostic and predictive biomarker in advanced prostate cancer. Expert Rev Mol Diagn 2018;18:155-63.

4. Galletti G, Leach BI, Lam L, et al. Mechanisms of resistance to systemic therapy in metastatic castrationresistant prostate cancer. Cancer Treat Rev 2017;57:16-27.

5. Luo J, Attard G, Balk SP, et al. Role of Androgen Receptor Variants in Prostate Cancer: Report from the 2017 Mission Androgen Receptor Variants Meeting. Eur Urol 2018;73:715-23.

6. Antonarakis ES, Lu C, Wang H, et al. AR-V7 and resistance to enzalutamide and abiraterone in prostate cancer. N Engl J Med 2014;371:1028-38.

7. Antonarakis ES, Lu C, Luber B, et al. Androgen Receptor Splice Variant 7 and Efficacy of Taxane Chemotherapy in Patients With Metastatic Castration-Resistant Prostate Cancer. JAMA Oncol 2015;1:582-91.

8. Armstrong AJ, Halabi S, Luo J, et al. Prospective Multicenter Validation of Androgen Receptor Splice Variant 7 and Hormone Therapy Resistance in High-Risk Castration-Resistant Prostate Cancer: The PROPHECY Study. J Clin Oncol 2019;37:1120-9.

9. Scher HI, Graf RP, Schreiber NA, et al. Phenotypic Heterogeneity of Circulating Tumor Cells Informs Clinical Decisions between AR Signaling Inhibitors and Taxanes in Metastatic Prostate Cancer. Cancer Res 2017;77:5687-98.

10. Halabi S, Lin CY, Kelly WK, et al. Updated prognostic model for predicting overall survival in first-line chemotherapy for patients with metastatic castrationresistant prostate cancer. J Clin Oncol 2014;32:671-7.

11. Del Re M, Crucitta S, Sbrana A, et al. AR-V7 and ARFL expression is associated with clinical outcome: a translational study in patients with castrate resistant prostate cancer. BJU Int 2019. [Epub ahead of print].

12. Antonarakis ES, Lu C, Luber B, et al. Clinical Significance of Androgen Receptor Splice Variant-7 mRNA Detection in Circulating Tumor Cells of Men With Metastatic Castration-Resistant Prostate Cancer Treated With Firstand Second-Line Abiraterone and Enzalutamide. J Clin Oncol 2017;35:2149-56.

Cite this article as: Chirra M, Petrioli R, Roviello G. Androgen receptor splice variant 7 in castration resistant prostate cancer treated with hormonal therapy: what is in the pipeline? Transl Cancer Res 2019;8(Suppl 6):S595-S597. doi: 10.21037/tcr.2019.08.34 